\title{
Synergistic growth inhibition by sorafenib and cisplatin in human osteosarcoma cells
}

\author{
QU YANG, SHANYONG ZHANG, MINGYANG KANG, RONGPENG DONG and JIANWU ZHAO \\ Department of Orthopedics, The Second Hospital of Jilin University, Changchun, Jilin 130042, P.R. China
}

Received December 10, 2014; Accepted January 19, 2015

DOI: $10.3892 / o r .2015 .3832$

\begin{abstract}
Molecular-targeted therapy has shown promise as a treatment for advanced osteosarcoma (OS). Sorafenib (SOR), a multikinase inhibitor, is the approved systemic drug of choice for OS, but has demonstrated limited benefits due to its toxicity and other adverse effects. Therapy strategies for reducing toxicity include using lower doses of SOR in combination with other complementary agents. Cisplatin (CDDP) has been shown to be a promising anticancer drug against various types of cancer including OS. In the present study, SOR was combined with CDDP to determine whether this combinatorial treatment suppressed tumor growth thereby simultaneously reducing doses of the two drugs for the treatment of OS. Human Saos-2 OS cells were treated with SOR and CDDP, alone and in combination, and the effect of these treatments on cell proliferation, colony formation, cell cycle, apoptosis, migration, and invasion, and involvement in receptor signaling, as well as tumor growth ability in nude mice was determined. It was found that the combination of low concentrations of SOR and CDDP significantly suppressed the cell proliferation, colony formation, migration and invasion, and induced cell apoptosis and cell cycle arrest in the G0/G1 stage, and suppressed tumor growth in a nude mouse model compared to the actions of either agent alone. The results also showed that SOR in combination with CDDP significantly suppressed the phosphorylation of extracellular signal-regulated kinase (ERK), which may contribute to the inhibition of tumor growth. These results suggested that SOR in combination with CDDP acts synergistically in the treatment of OS.
\end{abstract}

\section{Introduction}

Osteosarcoma (OS) is the most frequent primary solid malignancy of bone in children and adolescence $(1,2)$. The

Correspondence to: Professor Jianwu Zhao, Department of Orthopedics, The Second Hospital of Jilin University, No. 218 Ziqiang Street, Nanguan, Changchun, Jilin 130042, P.R. China

E-mail: zhaojiawu14208@sohu.com

Key words: sorafenib, cisplatin, osteosarcoma, tumor growth current therapy regimen for high-grade OS includes induction by neoadjuvant chemotherapy followed by surgical resection (mostly limb-sparing or rarely amputation), adjuvant chemotherapy and radiotherapy (3). However, significant advancements in the treatment of OS in recent years, and overall survival of OS patients has remained relatively constant for over two decades $(4,5)$. New, effective and welltolerated therapy strategies are therefore required to further improve the prognosis of OS patients.

Sorafenib (SOR) (Nexavar; Bayer HealthCare Pharmaceuticals-Onyx Pharmaceuticals) a multikinase inhibitor that targets Raf kinases as well as VEGFR-2/-3, PDGFR- $\beta$, Flt-3 and c-Kit, has been shown to exert potent tumor growth inhibition in vitro for various types of cancer $(6,7)$. Findings of a recent study confirmed that SOR inhibited the proliferation of the Saos-2 OS cell line and caused a series of biomolecule effects, including the change of VEGFR2 and extracellular signal-regulated kinase (ERK) gene expression, and the phosphorylation alteration of VEGFR2, RET, and MEK1 (8). Of note, a phase II trial explored SOR activity in patients with relapsed and unresectable OS and found that SOR demonstrated activity as a second- or third-line treatment in terms of progression-free survival at 4 months with some unprecedented long-lasting responses (5). However, the results of clinical studies have also indicated that treatment with SOR alone provides only minimal survival benefits for patients with advanced OS. In addition, SOR resulted in a variety of adverse reactions including diarrhea, hypertension and nausea $(9,10)$. It also caused cutaneous toxic effects including mucositis (20\%), rash (19-40\%), alopecia $(27 \%)$, xerosis $(16 \%)$, xerostomia $(11 \%)$, and hand-foot skin reaction (HFSR) (20-30\%) (11). One method for overcoming this toxicity is to use lower doses of SOR in combination with other complementary agents $(12,13)$. Therefore, the development of more efficacious combination therapies involving SOR and other agents appears to be an attractive approach for providing improved clinical outcomes in the treatment of OS. In a recent study it was demonstrated that combination therapies of SOR and other chemotherapeutic agents may result in synergistic or additive inhibitory effects on the growth of OS cells (14).

Cisplatin (CDDP) is an effective antitumor agent with a wide range of activity against various human solid tumors ovarian, bladder, cervical, head and neck, esophageal, small cell lung cancer (SCLC) and gastric cancer (15-18). CDDP 
was identified to have cytotoxic properties in the 1960 s, and earned a place as the key ingredient in the systemic treatment of germ cell cancers by the end of the 1970s. Generally, CDDP can form bivalent adducts with nucleophilic sites on purines in DNA, and yield predominantly DNA intra-strand cross links between adjacent purines (19). The use of CDDP treatment is considered a useful chemotherapeutic method for preoperative induction therapy for OS, with an improved survival rate (20). In addition, the inclusion of CDDP demonstrated improved outcome for patients with high-grade OS (21).

Although anti-OS activity has been shown for SOR and CDDP, to the best of our knowledge, the effect on OS of these drugs in combination has yet to be reported. We hypothesized that these drugs may synergize to be more effective than either agents administered independently. Therefore, in the present study, we determined whether the co-administration of low-dose SOR with CDDP may potentiate the inhibition of OS cell growth in vitro and in vivo.

\section{Materials and methods}

Cell culture. Human Saos-2 OS cells were purchased from the Cell Bank of Type Culture Collection of the Chinese Academy of Sciences, Shanghai Institute of Cell Biology, Chinese Academy of Sciences (Shanghai, China). The cells were cultured in Dulbecco's modified Eagle's medium (DMEM; Gibco-BRL, Grand Island, NY, USA) supplemented with $10 \%$ heat-inactivated fetal bovine serum (FBS; Gibco-BRL) at $37^{\circ} \mathrm{C}$ in a $5 \% \mathrm{CO}_{2}$ atmosphere and at $95 \%$ humidity.

Cell viability and colony formation. An MTT assay was used to determine the effect of different concentrations of CDDP and SOR alone or in combination on cell proliferation. Briefly, Saos-2 cells grown in monolayers were collected and dispensed in 96-well culture plates in $100 \mu 1$ of DMEM at a concentration of $5 \times 10^{3}$ cells/well. After $24 \mathrm{~h}$, different concentrations of SOR $(0-20 \mu \mathrm{M})$, CDDP $(0-10 \mu \mathrm{M})$, or both (0-10 $\mu \mathrm{M}$ SOX plus $5 \mu \mathrm{M}$ CDDP) were added to the cells. At the indicated time-points, $20 \mu \mathrm{l}$ of methylthiazole tetrazolium (MTT; Sigma-Aldrich, St. Louis, MO, USA) solution $(5 \mathrm{mg} / \mathrm{ml})$ was added into each well and cultured for $4 \mathrm{~h}$. Centrifugation $(12,000 \times \mathrm{g}, 2 \mathrm{~min})$ was performed to remove the supernatant, and $200 \mu \mathrm{l}$ of DMSO was added to each well followed by agitation for $10 \mathrm{~min}$ to dissolve the crystals. Absorbance was measured at $570 \mathrm{~nm}$ with a Microplate Reader (Molecular Devices Corp., Sunnyvale, CA, USA). This assay was performed in triplicate.

The effect of SOR or CDDP alone or in combination on the cell colony formation was assessed using a clonogenic assay. For this analysis, $1.0 \times 10^{3}$ cells were plated in 6-well plates in growth medium. Subsequent attaching overnight the cells were exposed to their respective half maximal inhibitory concentration $\left(\mathrm{IC}_{50}\right)$ values of SOR, CDDP, or their combinations or vehicle for $48 \mathrm{~h}$. The cells were then washed with drug-free medium and allowed to grow for 14 days in drug-free conditions. Colonies containing $>50$ cells were counted. Relative colony formation was determined by the ratio of the average number of colonies in treated cells to the average number of colonies in cells treated with solvent (DMSO). All the experiments were performed in triplicate.
Cell cycle and apoptotic assay. The effect of SOR or CDDP alone or in combination on cell cycle and apoptosis was examined by flow cytometry. Briefly, 5.0x $10^{5}$ Saos- 2 cells were plated in $60-\mathrm{mm}$ dishes and treated with their respective $\mathrm{IC}_{50}$ values of SOR, CDDP, or both for $48 \mathrm{~h}$. After treatment, the cells were collected and washed twice with PBS, fixed with $70 \%$ ethanol at $-20^{\circ} \mathrm{C}$ for $30 \mathrm{~min}$ and then stored at $4{ }^{\circ} \mathrm{C}$ overnight. Subsequently, the cells were washed with PBS again, treated with $100 \mathrm{ml}$ and $100 \mathrm{mg} / \mathrm{l} \mathrm{RNase}$ at $37^{\circ} \mathrm{C}$ for $30 \mathrm{~min}$, and stained with $100 \mathrm{ml}$ and $50 \mathrm{mg} / 1$ propidium iodide (PI; Sigma-Aldrich) at $4^{\circ} \mathrm{C}$ for $30 \mathrm{~min}$ in the dark. The multiplication cycle and apoptotic rate were measured by using Flow Cytometry (BD Biosciences, Mansfield, MA, USA), and the data were analyzed by CellQuest software (BD Biosciences San Jose, CA, USA).

In addition, caspase- $3,-8$ and -9 activity was detected as an additional indicator of apoptosis.

Caspase activity. Caspase- $3,-8$ and -9 activity was determined using Caspases Colorimetric Protease Assay kits (Millipore Corporation, Billerica, MA, USA) according to the manufacturer's instructions. Briefly, Saos-2 cells were treated with their respective $\mathrm{IC}_{50}$ values of SOR, CDDP, or both for $48 \mathrm{~h}$. After treatment, the cells were washed twice with ice-cold PBS and harvested by centrifugation. The cell pellets were then lysed in $150 \mu \mathrm{l}$ buffer provided in the kit. Protein concentrations of lysates were measured by the Lowry's method. An aliquot of lysates was incubated with $10 \mu \mathrm{l}$ substrate of their respective caspase at $37^{\circ} \mathrm{C}$ for $2 \mathrm{~h}$. The samples were measured in a Microplate Reader (Molecular Devices Corp.) at $405 \mathrm{~nm}$. The relative caspase-3, -8 and -9 activity of the control blank group was referred as 100 .

Wound-healing assay. To assess the effect of SOR or CDDP alone or in combination on cell migration, a wound-healing assay was performed. Briefly, $1 \times 10^{5} \mathrm{Saos}-2$ cells were plated in 12-well plates in DMEM containing 10\% FBS. After $24 \mathrm{~h}$, a scratch was made through the confluent cell monolayer, and the cells were treated with their respective $\mathrm{IC}_{50}$ values of $\mathrm{SOR}$, CDDP, or both in $3 \mathrm{ml}$ of complete DMEM medium. After a 48-h treatment, the cells were stained with hematoxylin and eosin (H\&E). The area of migration was observed under an Inverted Phase-Contrast Microscope (Leica DMR, Heidelberg, Germany). The experiments were performed in triplicate.

Transwell invasion assays. The invasion assays were performed using Transwell Insert Chambers (Corning, Inc., Corning, NY, USA). Briefly, Saos-2 cells were treated with their respective $\mathrm{IC}_{50}$ values of SOR, CDDP, or both for $48 \mathrm{~h}$. After treatment, $3 \times 10^{5}$ transfected cells were seeded into upper chambers pre-coated with Matrigel (BD Biosciences) in serum-free medium in triplicate. The medium containing $20 \%$ FBS in the lower chamber served as a chemoattractant. After culturing for $24 \mathrm{~h}$, the media were removed from the upper chamber by wiping with a cotton swab and the cells migrating to the lower surface of the filter were fixed in $70 \%$ ethanol for $30 \mathrm{~min}$ and stained with $0.2 \%$ crystal violet for $10 \mathrm{~min}$. The number of cells invading the Matrigel was counted in five randomly selected fields by an Inverted Microscope (Olympus, Tokyo, Japan). 
In addition, MMP-2 and-9 expression was determined by western blot analysis as an additional indicator of apoptosis.

Western blot analysis. Saos-2 cells were treated with their respective $\mathrm{IC}_{50}$ values of SOR, CDDP or their combination for $48 \mathrm{~h}$. Saos-2 cells were trypsinized, lysed in RIPA lysis buffer ( $\mathrm{pH} 7.4,150 \mathrm{mM} \mathrm{NaCl}, 0.1 \%$ SDS, $0.5 \%$ sodium deoxycholate, $1 \%$ NP-40 in PBS, protease complete inhibitor; Roche Diagnostics, Mannheim, Germany), frozen and thawed three times, then centrifuged $\left(13,000 \times \mathrm{g}, 20 \mathrm{~min}\right.$ at $\left.4^{\circ} \mathrm{C}\right)$ to remove insoluble material. The harvested supernatant served as the total proteins for the subsequent experiments. Total protein concentration was determined using the BCA Assay kit (Sigma-Aldrich). Cell extracts ( $20 \mu \mathrm{g}$ of protein) were separated by $8-15 \%$ SDS-PAGE gel and transferred to nitrocellulose membranes (Sigma-Aldrich). After blocking non-specific binding sites with 5\% dry milk in PBST, the membranes were incubated using the mouse monoclonal anti-human $\beta$-actin (1:5,000; Sigma-Aldrich), mouse monoclonal anti-human MMP-2 (1:2,000), mouse monoclonal anti-human MMP-9 $(1: 3,000)$, mouse monoclonal anti-human ERK $(1: 2,500)$ and mouse monoclonal anti-p-ERK $(1: 2,500)$ antibodies (all from Santa Cruz Biotechnology, Inc., Santa Cruz, CA, USA). The membranes were incubated with horseradish peroxidase-conjugated goat anti-mouse IgG (1:5,000; Santa Cruz Biotechnology, Inc.) for $2 \mathrm{~h}$ at room temperature. Protein bands were visualized with enhanced chemiluminescence reagent (ECL; Amersham, GE Healthcare, Velizy-Villacoublay, France). The blots were stripped and reprobed with anti- $\beta$-actin to control for loading variations. Quantity One software (Bio-Rad, Hercules, CA, USA) was used for quantification of protein bands

Tumor xenograft assay. To investigate the effects of SOR and CDDP alone or in combination on the tumorigenicity of xenograft and the influence on survival of tumor-burdened animals, 40 female BALB/c nude mice (aged 4-6 weeks) were obtained from the Experimental Animal Center of the Jilin University (Changchun, China). The research protocol was approved and mice were maintained in accordance with the Institutional Guidelines of the Experimental Animals of Jilin University.

Approximately 6- to 7-week-old female BALB mice were maintained under specific pathogen-free (SPF) conditions and provided with food and water ad libitum. The animals were fed with a normal pellet diet 1 week prior to the experimentation. Exponentially growing Saos- 2 cells were harvested and a tumorigenic dose of $2.5 \times 10^{6}$ cells was injected intraperitoneally into $\mathrm{BALB} / \mathrm{c}$ mice. When the tumor volume reached $100 \mathrm{~mm}^{3}$, the mice were divided randomly into 4 groups ( $\mathrm{n}=10 \mathrm{mice} / \mathrm{group}$ ). The control group received $1 \%$ polysorbate resuspended in deionized water. The remaining 3 groups were treated with CDDP (10 mg/kg body weight), SOX ( $80 \mathrm{mg} / \mathrm{kg}$ body weight), or CDDP plus SOX (5 and $40 \mathrm{mg} / \mathrm{kg}$ body weight, respectively) intraperitoneally on alternative days for 3 weeks. The doses were selected based on previously conducted experiments $(22,23)$. Tumor volume was measured before the treatment injections were administered and on the $7 \mathrm{th}, 14 \mathrm{th}$ and 21 st day of treatment. On day 22, the animals were euthanized using chloroform, tumor tissues were resected and the volume and weight measured.
In addition, spleen tissues were collected and cultured for a splenocyte surveillance study using an MTT assay as previously described (12).

Statistical analysis. Data are expressed as the mean \pm standard deviation (SD). Differences between groups were assessed by using one-way analysis of variance (ANOVA). Statistical analyses were undertaken using GraphPad Prism version 5.01 (GraphPad Software, San Diego, CA, USA) and the SPSS ${ }^{\circledR}$ statistical package, version 19.0 (SPSS Inc., Chicago, IL, USA) for Windows ${ }^{\circledR}$. $\mathrm{P}<0.05$ was considered as statistically significant.

\section{Results}

Effects of SOR and CDDP alone or in combination on cell proliferation and colony formation of Saos-2 cells. To assess the effect of SOR and CDDP alone or in combination on the viability of OS cells in vitro, Saos-2 cells were treated with different concentrations of SOR $(0-20 \mu \mathrm{M}), \operatorname{CDDP}(0-10 \mu \mathrm{M})$, or both (0-10 $\mu \mathrm{M}$ SOX plus $5 \mu \mathrm{M}$ CDDP) for $48 \mathrm{~h}$, and an MTT assay was performed. SOR inhibited cell viability dose-dependently with an $\mathrm{IC}_{50}$ of $5.5 \pm 0.16 \mu \mathrm{M}$ in Saos-2 cells (Fig. 1A). CDDP alone also reduced cell viability in a dose-dependent manner with an $\mathrm{IC}_{50}$ of $6.5 \pm 0.42 \mu \mathrm{M}$ in Saos-2 cells (Fig. 1B). The combinatorial treatment $(0-10 \mu \mathrm{M}$ in the presence of $5 \mu \mathrm{M}$ CDDP) resulted in a leftward shift of the concentration-response curve such that the $\mathrm{IC}_{50}$ values were reduced to $2.55 \pm 0.10 \mu \mathrm{M}$, indicating that treatment with SOR in combination with CDDP was more cytotoxic than the monotherapy groups. Based on the results we selected the respective $\mathrm{IC}_{50}$ values of drugs for further treatments throughout the study.

We examined whether the combination of relatively low concentrations of SOR and CDDP additively or synergistically inhibited Saos-2 cell proliferation with their respective $\mathrm{IC}_{50}$ values of SOR, CDDP or both at the indicated times. It was found that SOR and CDDP alone or in combination significantly inhibited cell proliferation compared to the control group (Fig. 1D, $\mathrm{P}<0.05$ ). The combinatorial treatment group significantly inhibited cell proliferation compared to the monotherapy groups $(\mathrm{P}<0.05$, Fig. 1D). No significant difference was identified between the SOR and CDDP groups ( $\mathrm{P}>0.05)$.

The effects of SOR and CDDP alone or in combination on the colony formation of Saos-2 cells were also analyzed. Compared with the control group, colony number of tumor cella was significantly reduced in SOR and CDDP alone or in the combinatorial groups $(\mathrm{P}<0.05$, Fig. $1 \mathrm{E}$ and $\mathrm{F})$. SOR in combination with CDDP resulted in an even higher percentage of reduction than the higher doses of either drug alone $(\mathrm{P}<0.05$, Fig. 1E and F).

Effects of SOR and CDDP alone or in combination on cell cycle and apoptosis of Saos-2 cells. The effects of SOR and CDDP alone or in combination on the cycles of Saos-2 cells were analyzed by flow cytometry. It was found that Saos-2 cells treated with SOR and CDDP alone or in combination had an increased percentage of arrest at the G0/G1 phase compared with the control group (Fig. 2A and B). The combinatorial treatment resulted in an even greater percentage of 
A

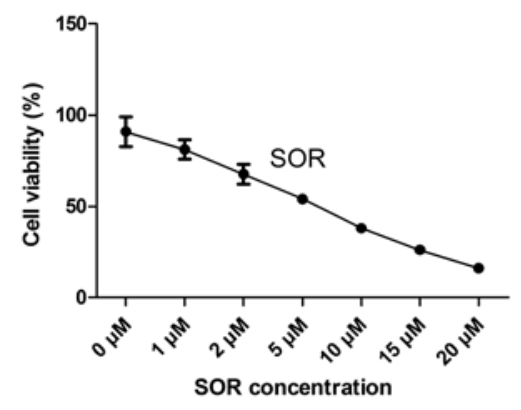

D

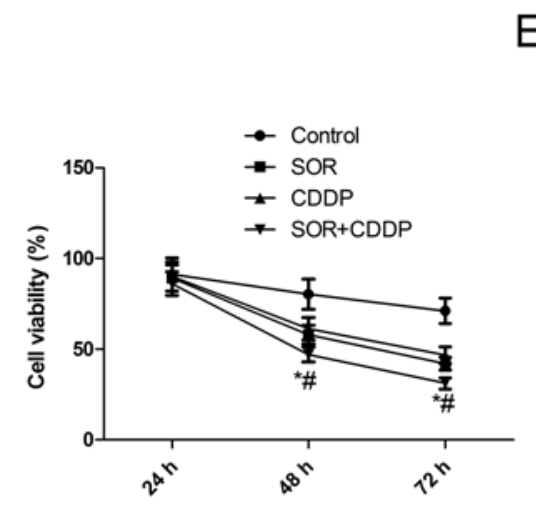

B

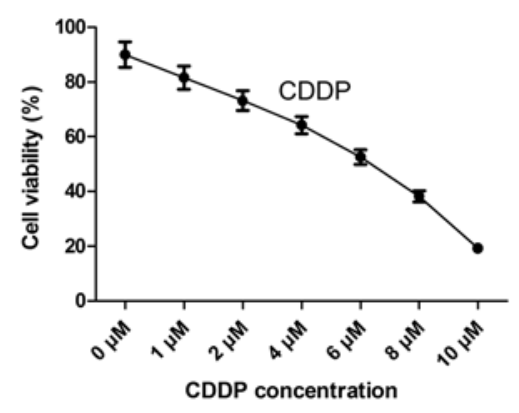

$\mathrm{E}$

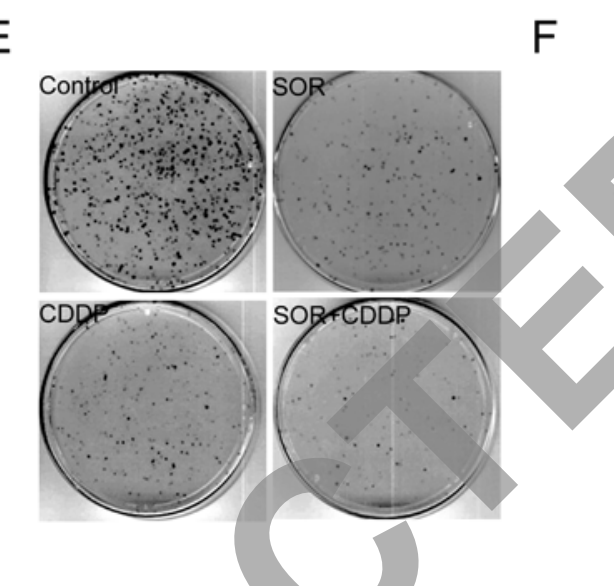

C

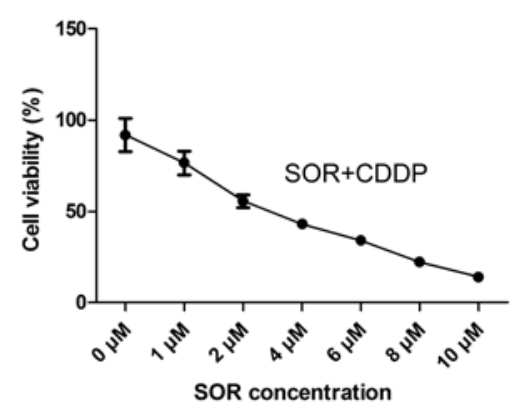

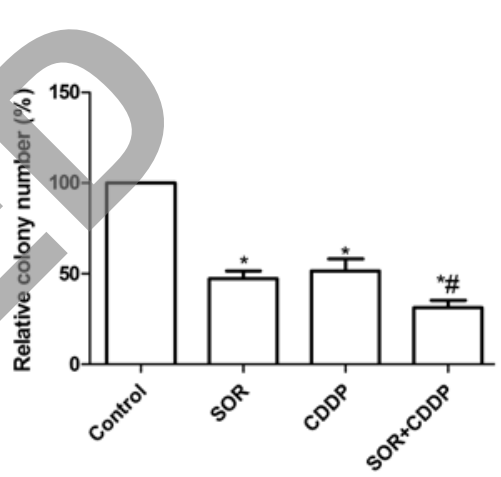

Figure 1. Effect of sorafenib (SOR) and cisplatin (CDDP) alone or in combination on the proliferation and colony formation of Saos-2 cells. In vitro viability assay of Saos- 2 cells treated with (A) SOR $(0-20 \mu \mathrm{M})$, (B) CDDP $(0-10 \mu \mathrm{M})$, or (C) both $(0-10 \mu \mathrm{M}$ SOX plus $5 \mu \mathrm{M}$ CDDP) for $72 \mathrm{~h}$. (D) Saos- 2 cells were treated with their $\mathrm{IC}_{50}$ concentration of SOR and CDDP individually and their combinations at the indicated time points. (E and F) Clone formation of Saos-2 cells was photographed, and counted following treatment with SOR and CDDP alone or in combination. Data are expressed as the means \pm SD of three independent experiments. ${ }^{*} \mathrm{P}<0.05$ vs. control, ${ }^{\#} \mathrm{P}<0.05$ vs. SOR alone.

arrest at the G0/G1 phase than the higher doses of either drug alone $(\mathrm{P}<0.01)$.

To investigate whether SOR and CDDP alone or in combination induced apoptosis, a cell apoptosis assay was performed by flow cytometry. Flow cytometry revealed that treatment with SOR and CDDP alone or in combination led to a marked increase in apoptotic cells compared to the control group $(\mathrm{P}<0.05)$ as shown Fig. $2 \mathrm{C}$. In addition, treatment with the combination of SOR and CDDP resulted in a marked increase in apoptotic cells compared to the monotherapy group.

To examine the possible mechanism of the pro-apoptotic effect of combination with SOR and CDDP, caspase-3, -8 and -9 activity was detected using ELISA. The results showed that caspase-3, -8 and -9 activity was markedly increased in the SOR and CDDP alone or combination treatment groups compared to the control group ( $\mathrm{P}<0.05$; Fig. 2D-F). Compared to the single-drug treatment group, the combinatorial treatment significantly increased caspase- $3,-8$ and -9 activity $(\mathrm{P}<0.05$; Fig. 2D-F).

Effects of SOR and CDDP alone or in combination on the migration and invasion apoptosis of Saos-2 cells. To ascertain the inhibitory effect of SOR and CDDP as a single or combined treatment on breast cancer migration, a woundhealing assay was performed to investigate the effects on the migration potential of Saos- 2 cells. After $24 \mathrm{~h}$ treatment, cells in the SOR and CDDP alone or combinatorial group migrated significantly less than those in the control group $(\mathrm{P}<0.05$,
Fig. 3A and B). Cell migration in the combination group was lower than either single drug alone $(\mathrm{P}<0.05$, Fig. $3 \mathrm{~A}$ and $\mathrm{B})$.

The ability of SOR and CDDP alone or in combination to reduce the invasiveness of Saos-2 cells was further investigated by the Transwell system assay. It was found that invasion was also decreased significantly with SOR and CDDP alone or in the combination treatment groups compared to the control group (Fig. 3C and D). The result of the cell invasiveness assay showed that there was no significant difference in the number of cells that had passed through the simulated basement membrane between the SOR and CDDP groups. Compared with the results with either agent alone, the combination of SOR and CDDP greatly inhibited the invasion of Saos- 2 cells.

To determine the potential mechanism of SOR in combination with CDDP inhibition cell migration and invasion in vitro, the invasion associated with MMP-2 and -9 protein expression was determined by western blot analysis. Results of the analysis revealed a significant decrease in MMP-2 and -9 proteins in the SOR and CDDP alone or combination group compared to the control group $(\mathrm{P}<0.05$, Fig. $3 \mathrm{E}$ and $\mathrm{F})$. The combination group obviously decreased the MMP-2 and -9 protein expression compared to the monotherapy groups $(\mathrm{P}<0.05$, Fig. 3E and F).

Effects of SOR and CDDP on MEK/ERK signaling pathway in Saos-2 cells. The effects of SOR are known to be transduced by the MEK/ERK signaling pathway (24). Results of a recent study demonstrated that this pathway may be involved in the 
A
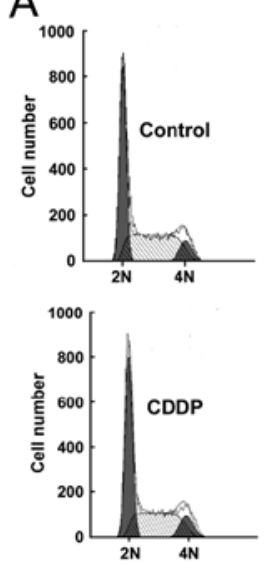

B
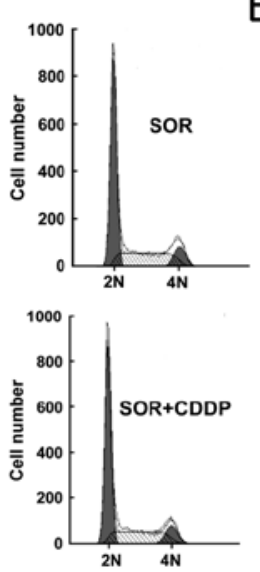

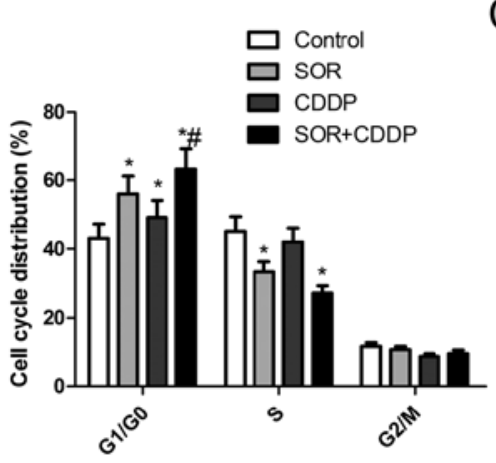

C

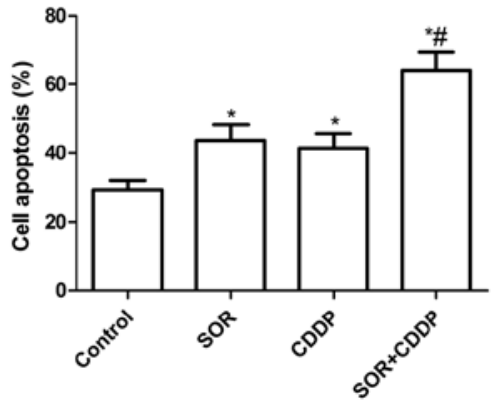

$E$

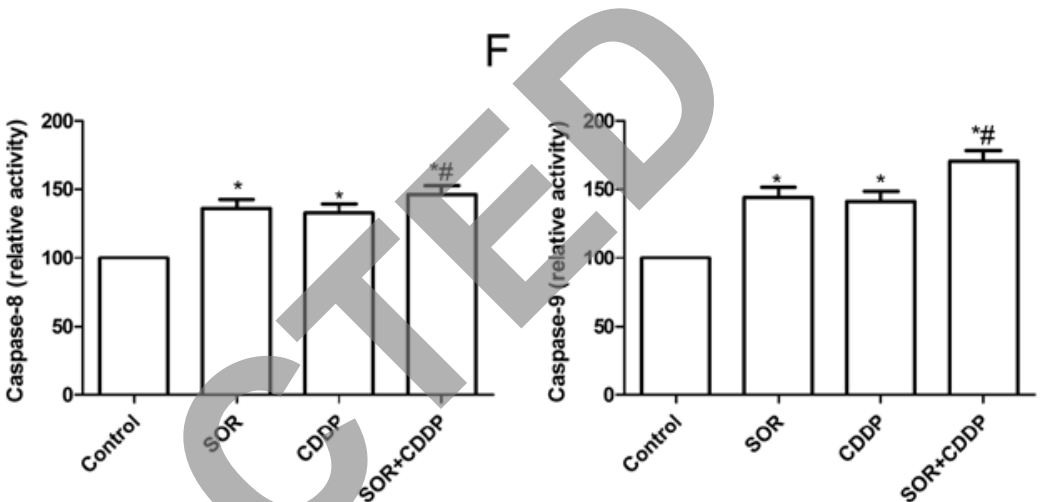

Figure 2. Effect of sorafenib (SOR) and cisplatin (CDDP) alone or in combination on the cell cycle and apoptosis of Saos-2 cells. (A) Cell DNA content distribution in each phase after treatment with SOR and CDDP alone or in combination. (B) Percentage of cells distributed in each phase of the cell cycle. (C) Apoptosis of Saos-2 cells was determined following treatment with SOR and CDDP alone or in combination. (D) Caspase-3, (E) caspase-8 and (F) caspase-9 activity were determined following treatment with SOR and CDDP alone or in combination. Data are expressed as the means \pm SD of three independent experiments. ${ }^{*} \mathrm{P}<0.05$ vs. control, ${ }^{~} \mathrm{P}<0.05$ vs. SOR alone.

A
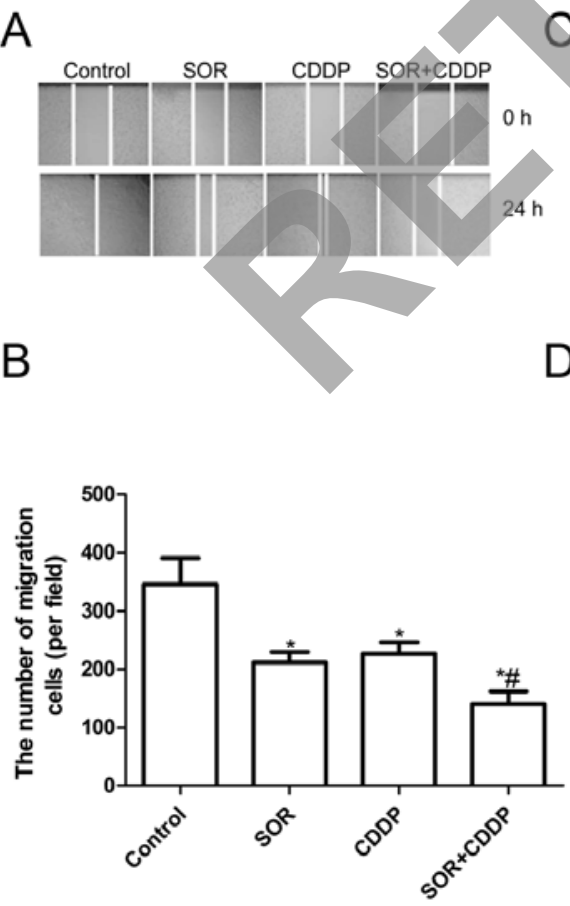

C

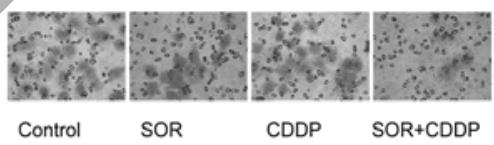

D
E

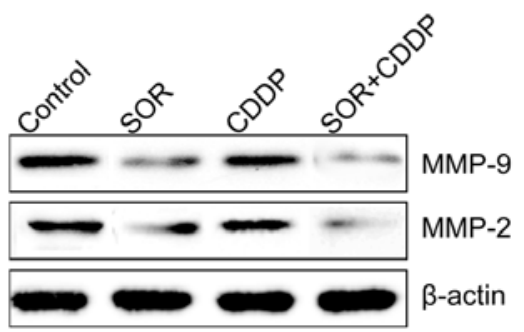

$\mathrm{F}$

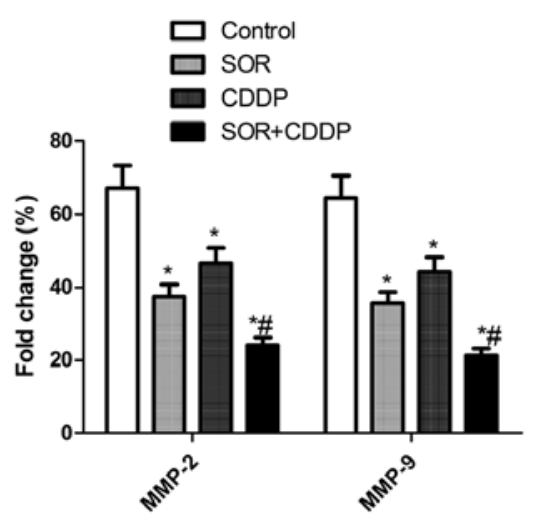

Figure 3. Effect of sorafenib (SOR) and cisplatin (CDDP) alone or in combination on the migration and invasion of Saos-2 cells. (A) Cell migration was determined by wound-healing assay following treatment with SOR and CDDP alone or in combination. (B) The number of migration cells was counted. (C) Cell invasion was determined by Matrigel Transwell following treatment with SOR and CDDP alone or in combination. (D) The number of invasion cells was counted. (E) Western blot analysis of MMP-2 and MMP-9 protein expression following treatment with SOR and CDDP alone or combination. $\beta$-actin was used as an internal control. (F) Relative quantification of MMP-2 and MMP-9 protein by densitometric analysis, ${ }^{*} \mathrm{P}<0.05$ vs. control, ${ }^{\text {}} \mathrm{P}<0.05$ vs. SOR alone. 

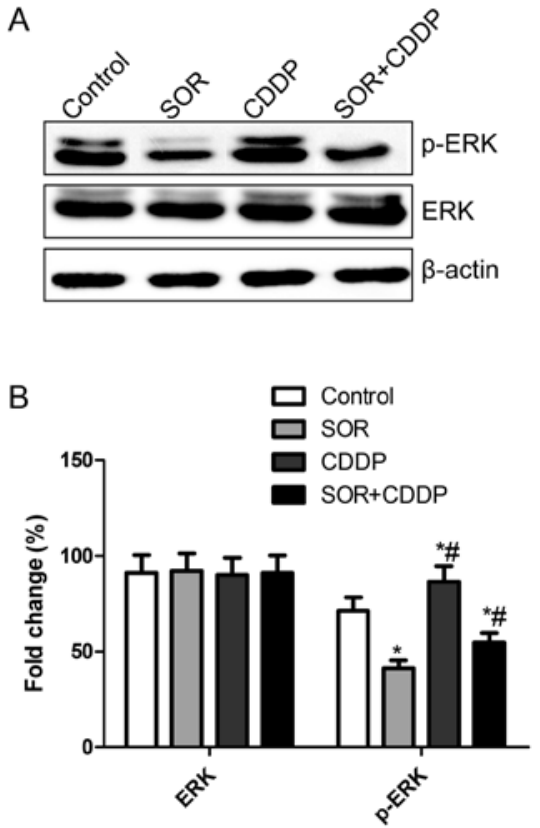

Figure 4. Effect of sorafenib (SOR) and cisplatin (CDPP) alone or combination on MEK/ERK signaling in Saos-2 cells. (A) Cells were treated with SOR and CDPP alone or both for $4 \mathrm{~h}$. Western blot analysis were performed using specific antibodies against the indicated proteins. Blots were reprobed for $\beta$-actin to normalize each lane for protein content. (B) Relative quantification of ERK and p-ERK protein by densitometric analysis, ${ }^{*} \mathrm{P}<0.05$ vs. control, ${ }^{\#} \mathrm{P}<0.05$ vs. SOR alone.

SOR effect on OS cells (25). As the combination of SOR and CDDP showed the greatest inhibition of cell proliferation at low SOR and CDDP concentrations, insight was gained with regard to the mechanism. Therefore, we evaluated the effect of SOR and CDDP alone and in combination on the expression ERK and phosphorylation-ERK (p-ERK) by western blotting $4 \mathrm{~h}$ after treatment with their respective $\mathrm{IC}_{50}$ values of SOR and CDDP alone and in combination. Treatment with SOR alone reduced p-ERK in Saos-2 cells (Fig. 4), while treatment with CDDP alone led to marked addition of p-ERK expression in Saos-2 cells (Fig. 4). Expression of p-ERK was lower in SOR in combination with CDDP compared with the control, although it did not reach the effect of SOR alone. In addition, total ERK expression did not alter in all the groups.

Antitumor activity of SOR and CDDP alone or in combination in nude mouse-bearing Saos-2 cells. We assessed the in vivo therapeutic efficacy of SOR and CDDP alone or in combination in female BALB mouse-bearing Saos-2 tumor cells. Tumors were monitored every 7 days from the time that they became evident. Tumor volume following treatment with SOR and CDDP alone or in combination was significantly reduced for tumor cells compared with the control group ( $\mathrm{P}<0.05$, Fig. $5 \mathrm{~A}$ and $\mathrm{B}$ ). Treatment with the combination of SOR and CDDP resulted in marked inhibition of tumor growth compared to the monotherapy groups $(\mathrm{P}<0.05$, Fig. 5A and B). Twenty two days after implantation, the animals were sacrificed and tumor weights were measured. The results showed that the tumor weight of SOR and CDDP alone or in combination was reduced compared to that of the control group $(\mathrm{P}<0.05$, Fig. $5 \mathrm{~A}$ and $\mathrm{C})$. The combination group greatly inhibited tumor growth compared to the monotherapy group $(\mathrm{P}<0.05$, Fig. $5 \mathrm{~A}$ and $\mathrm{C})$. We assessed the efficacy of SOR and CDDP alone or in combination in modulating splenocyte cell proliferation using an MTT
B

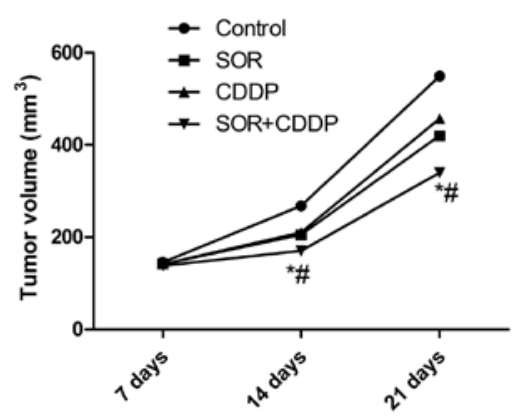

C

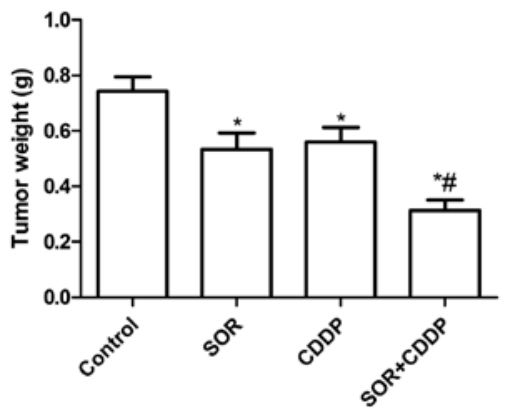

D

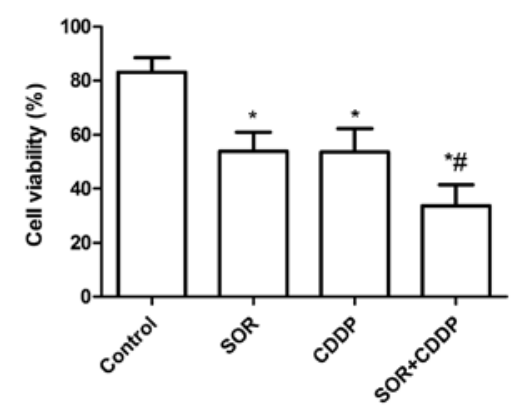

Figure 5. Antitumor activity of sorafenib (SOR) and cisplatin (CDPP) alone or combination in BALB/c mice bearing Saos-2 cells. (A) Images of tumor tissue from different treatments collected after sacrifice at day 22. (B) Tumor volume in treated and untreated mice at 7 th, 14th and 21th day. (C) Tumor weight in treated and untreated mice for 22 days. (D) MTT assay of proliferation of splenocytes from mice. Data are expressed as are the means \pm SD. ${ }^{*} \mathrm{P}<0.05$ vs. control, ${ }^{\#} \mathrm{P}<0.05$ vs. SOR alone. 
assay. It was found that SOR and CDDP alone or in combination significantly decreased cell proliferation compared to the control group $(\mathrm{P}<0.05$, Fig. 5D). The treatment group markedly decreased cell proliferation compared to the monotherapy groups $(\mathrm{P}<0.05$, Fig. 5D). These results indicated that SOR in combination with CDDP treatment markedly suppresses their tumorigenicity in nude mice.

\section{Discussion}

In the present study, we have provided convincing evidence that SOR in combination with CDDP enhanced the anti-proliferative and pro-apoptotic effects on OS cells in vitro and suppressed tumor growth of OS in a nude mouse model allowing for the use of lower doses of SOR and CDDP than those currently used. Although the two compounds have each been extensively studied, to the best of our knowledge, the present study is the first to show that combining a clinically applied therapy in SOR and CDDP may inhibit OS tumor growth in vitro and in vivo.

Chemotherapeutic drugs are most effective when administered in combination (combined chemotherapy) due to involvement of the combined chemotherapeutic drugs with different mechanisms of action, which contribute to decrease the possibility of drug resistance to cancer cella and reduction of the dose of the chemotherapeutic drugs being used. In addition, drugs are combined with different effects, and each drug can be used at its optimal dose, without intolerable side effects (26). SOR is safe and effective in patients with unresectable hepatocellular carcinoma (HCC) and has received FDA approval for this indication. Recent studies have shown that SOR alone significantly inhibited the growth of OS cells, and its combinations with other drugs increased the inhibitory effect on OS cells (14). CDDP is one of the most common first-line chemotherapeutic drugs for OS because of its DNA cross-linking activity (27). However, not all OS patients are sensitive to CDDP treatment (28). Accumulating evidence has shown that CDDP in combination with other drugs, such as methotrexate and pemetrexed, significantly inhibited OS cells growth in vitro and in vivo $(29,30)$. Of note, the synergistic anti-proliferative and pro-apoptotic effects of SOR in combination with CDDP were confirmed in human liver and gastric cancer cells $(18,31)$. Although SOR and CDDP alone or in combination have been found to inhibit other types of tumor growth, the effect on OS of SOR in combination CDDP has, to the best of our knowledge, yet to be reported. Results of the present study have demonstrated that SOR and CDDP inhibited the proliferation, migration and invasion of OS cells in vitro and suppressed the tumor growth of OS in a nude mouse model compared to SOR or CDDP, demonstrating synergistic effects. Although the results are encouraging, the antitumor effects of this combination should be investigated in OS patients in future studies.

The MEK/ERK signaling pathway plays a critical role in cell cycle, apoptosis proliferation and differentiation, and is an important downstream pathway of angiogenesis. ERK and its upstream kinase MEK localize to the extra-luminal face of autophagosomes and ERK phosphorylation is upregulated by lipidation of autophagic protein LC3 (32). It has been shown that the treatment of various types of cancer, such as ovarian carcinoma (33), melanoma (34), and liver cancer cells (35) with CDDP caused ERK activation and cell death, and this latter effect was potentiated by ERK inhibitors, indicating that ERKs behave as survival-inducing kinases in these cells. Consistent with those results, our study has shown that CDDP caused ERK activation in Saos-2 cells. In addition, blockade of ERK activation, either by pharmacological inhibitors or gene transfer procedures, reduced the CDDP toxicity in various types of cancer, such as cervical carcinoma (36), hepatoblastoma (37), OS and neuroblastoma (38) cell lines. SOR, an oral small-molecule multikinase inhibitor, developed by high-throughput screening of massive libraries of synthetic compounds primarily as a RAF (ras-activated factor) inhibitor blocking the RAF/MEK/ERK1/2 pathway, has been widely used to treat various cancer types. In the present study, we found that SOR in combination with CDDP treatment resulted in a marked decrease of phosphorylated ERK compared to the CDDP group, without altering the total protein levels of ERK each group, suggesting that SOR decreased CDDP toxicity in OS cells, at least in part by inhibiting the MEK/ERK pathway.

In summary, the findings of the present study suggest that, SOR in combination with CDDP was able to significantly inhibit cell proliferation, colony formation, migration and invasion, and induce cell apoptosis and arrest in the G0/ G1 stage in vitro, as well as suppress tumor growth in vivo, compared to SOR or CDDP alone, demonstrating a synergistic effect. In addition, this combination inhibited the MEK/ERK signaling pathway, which contributes to inhibition of tumor growth and reduction of CDDP toxicity to Saos- 2 cells. These findings suggest that the combination of SOR and CDDP is a promising drug candidate for the treatment of OS.

\section{Acknowledgements}

This study was supported by the Science and Technology Research and Innovation Team funded of Jilin provincial (JL20130518).

\section{References}

1. Ottaviani G, Robert RS, Huh WW, Palla S and Jaffe N: Sociooccupational and physical outcomes more than 20 years after the diagnosis of osteosarcoma in children and adolescents: limb salvage versus amputation. Cancer 119: 3727-3736, 2013.

2. Ottaviani $\mathrm{G}$ and Jaffe N: The epidemiology of osteosarcoma. Cancer Treat Res 152: 3-13, 2009.

3. Ta HT, Dass CR, Choong PF and Dunstan DE: Osteosarcoma treatment: state of the art. Cancer Metastasis Rev 28: 247-263, 2009

4. Chou AJ, Kleinerman ES, Krailo MD, et al: Addition of muramyl tripeptide to chemotherapy for patients with newly diagnosed metastatic osteosarcoma: a report from the Children's Oncology Group. Cancer 115: 5339-5348, 2009.

5. Grignani G, Palmerini E, Dileo P, et al: A phase II trial of sorafenib in relapsed and unresectable high-grade osteosarcoma after failure of standard multimodal therapy: an Italian Sarcoma Group study. Ann Oncol 23: 508-516, 2012.

6. Wilhelm SM, Carter C, Tang L, et al: BAY 43-9006 exhibits broad spectrum oral antitumor activity and targets the $\mathrm{RAF} / \mathrm{MEK} / \mathrm{ERK}$ pathway and receptor tyrosine kinases involved in tumor progression and angiogenesis. Cancer Res 64: 7099-7109, 2004.

7. Liu L, Cao Y, Chen C, et al: Sorafenib blocks the RAF/MEK/ERK pathway, inhibits tumor angiogenesis, and induces tumor cell apoptosis in hepatocellular carcinoma model PLC/PRF/5. Cancer Res 66: 11851-11858, 2006. 
8. Mei J, Zhu X, Wang Z and Wang Z: VEGFR, RET, and RAF/MEK/ERK pathway take part in the inhibition of osteosarcoma MG63 cells with sorafenib treatment. Cell Biochem Biophys 69: 151-156, 2014.

9. Almhanna K and Philip PA: Safety and efficacy of sorafenib in the treatment of hepatocellular carcinoma. Onco Targets Ther 2 261-267, 2009.

10. Strumberg D, Clark JW, Awada A, et al: Safety, pharmacokinetics, and preliminary antitumor activity of sorafenib: a review of four phase I trials in patients with advanced refractory solid tumors. Oncologist 12: 426-437, 2007.

11. Lacouture ME, Reilly LM, Gerami P and Guitart J: Hand foot skin reaction in cancer patients treated with the multikinase inhibitors sorafenib and sunitinib. Ann Oncol 19: 1955-1961, 2008.

12. Zhang H, Li Z and Wang K: Combining sorafenib with celecoxib synergistically inhibits tumor growth of non-small cell lung cancer cells in vitro and in vivo. Oncol Rep 31: 1954-1960, 2014

13. Morisaki T, Umebayashi M,Kiyota A, et al: Combining celecoxib with sorafenib synergistically inhibits hepatocellular carcinoma cells in vitro. Anticancer Res 33: 1387-1395, 2013.

14. Pignochino Y, Dell'Aglio C, Basirico M, et al: The combination of sorafenib and everolimus abrogates mTORC1 and mTORC2 upregulation in osteosarcoma preclinical models. Clin Cancer Res 19: 2117-2131, 2013.

15. Ma JJ, Chen BL and Xin XY: XIAP gene downregulation by small interfering RNA inhibits proliferation, induces apoptosis, and reverses the cisplatin resistance of ovarian carcinoma. Eur J Obstet Gynecol Reprod Biol 146: 222-226, 2009.

16. Cheng JQ, Jiang X, Fraser M, et al: Role of X-linked inhibitor of apoptosis protein in chemoresistance in ovarian cancer: possible involvement of the phosphoinositide-3 kinase/Akt pathway. Drug Resist Updat 5: 131-146, 2002.

17. Holt SV, Brookes KE, Dive C and Makin GW: Downregulation of XIAP by AEG35156 in paediatric tumor cells induces apoptosis and sensitises cells to cytotoxic agents. Oncol Rep 25: 1177-1181, 2011.

18. Tao $\mathrm{C}$, Lin $\mathrm{H}$ and Chen S: The regulation of ERK and p-ERK expression by cisplatin and sorafenib in gastric cancer cells. Gene 552: 106-115, 2014

19. Yuan JM, Li XD, Liu ZY, et al: Cisplatin induces apoptosis via upregulating Wrap53 in U-2OS osteosarcoma cells. Asian Pac J Cancer Prev 12: 3465-3469, 2011.

20. Abe S, Nishimoto Y, Isu K, Ishii T and Goto T, Japanese Musculoskeletal Oncology Group: Preoperative cisplatin for initial treatment of limb osteosarcoma. its local effect and impact on prognosis. Cancer Chemother Pharmacol 50: 320-324, 2002

21. Anninga JK, Gelderblom H, Fiocco M, et al: Chemotherapeutic adjuvant treatment for osteosarcoma: where do we stand? Eur J Cancer 47: 2431-2445, 2011.

22. Zhao JG, Ren KM and Tang J: Overcoming 5-Fu resistance in human non-small cell lung cancer cells by the combination of 5-Fu and cisplatin through the inhibition of glucose metabolism. Tumour Biol 35: 12305-12315, 2014.

23. Murakami M, Zhao S, Zhao Y, et al: Increased intratumoral fluorothymidine uptake levels following multikinase inhibitor sorafenib treatment in a human renal cell carcinoma xenograft model. Oncol Lett 6: 667-672, 2013
24. Gollob JA, Wilhelm S, Carter C and Kelley SL: Role of Raf kinase in cancer: therapeutic potential of targeting the Raf/MEK/ERK signal transduction pathway. Semin Oncol 33: 392-406, 2006

25. Walter I, Wolfesberger B, Miller I, et al: Human osteosarcoma cells respond to sorafenib chemotherapy by downregulation of the tumor progression factors S100A4, CXCR4 and the oncogene FOS. Oncol Rep 31: 1147-1156, 2014.

26. Ullah MF: Cancer multidrug resistance (MDR): a major impediment to effective chemotherapy. Asian Pac J Cancer Prev 9: 1-6, 2008.

27. Meyers PA, Schwartz CL, Krailo M, et al: Osteosarcoma: a randomized, prospective trial of the addition of ifosfamide and/or muramyl tripeptide to cisplatin, doxorubicin, and high-dose methotrexate. J Clin Oncol 23: 2004-2011, 2005.

28. Bruheim S, Xi Y, Ju J and Fodstad O: Gene expression profiles classify human osteosarcoma xenografts according to sensitivity to doxorubicin, cisplatin, and ifosfamide. Clin Cancer Res 15: 7161-7169, 2009

29. Yu WX, Tang LN, Lin F, Yao Y and Shen Z: Comparison of pemetrexed plus cisplatin with gemcitabine plus docetaxel in refractory/metastatic osteosarcoma: clinical outcomes from a retrospective database monitored in a single institute. Oncol Lett 8: 2243-2248, 2014

30. Lin F, Wang Q, Yu W, et al: Clinical analysis of Chinese limb osteosarcoma patients treated by two combinations of methotrexate, cisplatin, doxorubicin and ifosfamide. Asia Pac J Clin Oncol 7: 270-275, 2011.

31. WeiY, Shen N, Wang Z, et al: Sorafenib sensitizes hepatocellular carcinoma cell to cisplatin via suppression of Wnt/ $\beta$-catenin signaling. Mol Cell Biochem 381: 139-144, 2013.

32. Martinez-Lopez N, Athonvarangkul D, Mishall P, Sahu S and Singh R: Autophagy proteins regulate ERK phosphorylation. Nat Commun 4: 2799, 2013.

33. Hayakawa J, Ohmichi M, Kurachi H, et al: Inhibition of extracellular signal-regulated protein kinase or c-Jun N-terminal protein kinase cascade, differentially activated by cisplatin, sensitizes human ovarian cancer cell line. J Biol Chem 274: 31648-31654, 1999.

34. Mandic A, Viktorsson K, Heiden T, Hansson J and Shoshan MC The MEK1 inhibitor PD98059 sensitizes C8161 melanoma cells to cisplatin-induced apoptosis. Melanoma Res 11: 11-19, 2001.

35. Eicher C, Dewerth A, Thomale J, et al: Effect of sorafenib combined with cytostatic agents on hepatoblastoma cell lines and xenografts. Br J Cancer 108: 334-341, 2013.

36. Wang X, Martindale JL and Holbrook NJ: Requirement for ERK activation in cisplatin-induced apoptosis. J Biol Chem 275: 39435-39443, 2000.

37. Yeh PY, Chuang SE, Yeh KH, Song YC, Ea CK and Cheng AL: Increase of the resistance of human cervical carcinoma cells to cisplatin by inhibition of the MEK to ERK signaling pathway partly via enhancement of anticancer drug-induced NF kappa B activation. Biochem Pharmacol 63: 1423-1430, 2002.

38. Woessmann W, Chen X and Borkhardt A: Ras-mediated activation of ERK by cisplatin induces cell death independently of p53 in osteosarcoma and neuroblastoma cell lines. Cancer Chemother Pharmacol 50: 397-404, 2002. 